\title{
Atlas of STIS-HST spectra of Seyfert galaxies
}

\author{
Patrícia F. Spinelli ${ }^{1}$, C. H. Brandt ${ }^{1}$, \\ T. Storchi-Bergmann ${ }^{1}$ and D. Calzetti ${ }^{2}$ \\ ${ }^{1}$ Instituto de Física, UFRGS, Brazil email: pat@if.ufrgs.br \\ ${ }^{2}$ STScI, USA email: calzetti@stsci.edu
}

\begin{abstract}
We announce the construction of an Atlas of STIS-HST Spectra of Seyfert Galaxies, covering the UV and/or optical spectral range. Nuclear spectra of these galaxies have been extracted in windows of width $0.2^{\prime \prime}$ for an optimized sampling (as this is the width of the slit in most cases), from a long-slit spectrum usually covering $52^{\prime \prime}$. Extranuclear spectra have also been extracted, usually using larger windows to improve signal-to-noise ratio. Spectra obtained with different gratings have been combined in order to provide the largest possible wavelength coverage for each galaxy.
\end{abstract}

\section{Introduction}

When using the HST archive to recover spectra of Seyfert galaxies (which are the objects of our interest), one usually obtains several pieces of spectra covering different spectral ranges. Besides extracting the desired spectra, one usually needs to combine them after correcting for the bad signal at the borders. After having made this work for several Seyferts, we decided to extend this work to all Seyferts with STIS spectra in the HST archive and make these spectra available through the internet.

\section{Methodology}

The initial sample of Seyferts was selected from the Veron Catalog, as all Seyfert galaxies with redshifts $z<1.3$, comprising 80 galaxies. We began by extracting onedimensional spectra from the long-slit spectra. When more than one exposure for each spectral range (and with the same spectral resolution and orientation) was available, we constructed averages to improve the signal-to-noise ratio, eliminating also cosmicrays and other defects when detected. The final spectra was obtained by combining the spectra covering different spectral ranges. A table is being constructed containing both information on the final spectra (e.g. spectral range, number of combined spectra, spectral resolution), as well as on the individual spectra combined, such as exposure times and wavelength ranges.

\section{Results}

A preliminary and partial version of the tabulated information about individual and final spectra is presented in Table 1. Sample of final spectra of the galaxies are shown in Fig 1.

This is an on-going project. The total number of galaxies is now 50, for wich we have nuclear and extranuclear spectra. The table and the spectra will be available through the internet in the address:

wWw.stsci.edu/ftp/catalogs/nearby_galaxies . 
Combined Spectra $\mid$ Individual Spectra

\begin{tabular}{|c|c|c|c|c|c|c|c|}
\hline Galaxy & $\lambda_{i}$ & $\lambda_{f}$ & $N S$ & $\lambda_{i}$ & $\lambda_{f}$ & Exposure Time & $R$ \\
\hline \multirow[t]{5}{*}{ NGC4501 } & 2900 & 6867 & 5 & 6295 & 6867 & $900 \mathrm{~s}$ & 6581 \\
\hline & & & & 6295 & 6867 & $933 \mathrm{~s}$ & 6581 \\
\hline & & & & 6295 & 6867 & $840 \mathrm{~s}$ & 6581 \\
\hline & & & & 2900 & 5700 & $840 \mathrm{~s}$ & 4300 \\
\hline & & & & 2900 & 5700 & $804 \mathrm{~s}$ & 4300 \\
\hline \multirow[t]{2}{*}{ MARK348 } & 2900 & 5700 & 2 & 4950 & 5236 & $1410 \mathrm{~s}$ & 9090 \\
\hline & & & & 2900 & 5700 & $600 \mathrm{~s}$ & 800 \\
\hline \multirow[t]{8}{*}{ NGC1097 } & 1140 & 10266 & 8 & 1140 & 1730 & $2048 \mathrm{~s}$ & 1190 \\
\hline & & & & 1140 & 1730 & $2947 \mathrm{~s}$ & 1190 \\
\hline & & & & 1140 & 1730 & $2947 \mathrm{~s}$ & 1190 \\
\hline & & & & 1568 & 3184 & $2048 \mathrm{~s}$ & 740 \\
\hline & & & & 1568 & 3184 & $2947 \mathrm{~s}$ & 740 \\
\hline & & & & 1568 & 3184 & $2947 \mathrm{~s}$ & 740 \\
\hline & & & & 2900 & 5700 & $1008 \mathrm{~s}$ & 800 \\
\hline & & & & 5236 & 10266 & $936 \mathrm{~s}$ & 790 \\
\hline \multirow[t]{3}{*}{ NGC5252 } & 2900 & 6867 & 3 & 6295 & 6867 & $1080 \mathrm{~s}$ & 5980 \\
\hline & & & & 6295 & 6867 & $970 \mathrm{~s}$ & 5980 \\
\hline & & & & 2900 & 5700 & $600 \mathrm{~s}$ & 800 \\
\hline
\end{tabular}

Table 1. Partial table with relevant information on the spectra where: $\lambda_{i}$ and $\lambda_{f}$ are inicial and final wavelengths in angstrons, $N S$ is the total number of individual spectra combined to obtain the final spectrum and $R$ is the spectral resolution.

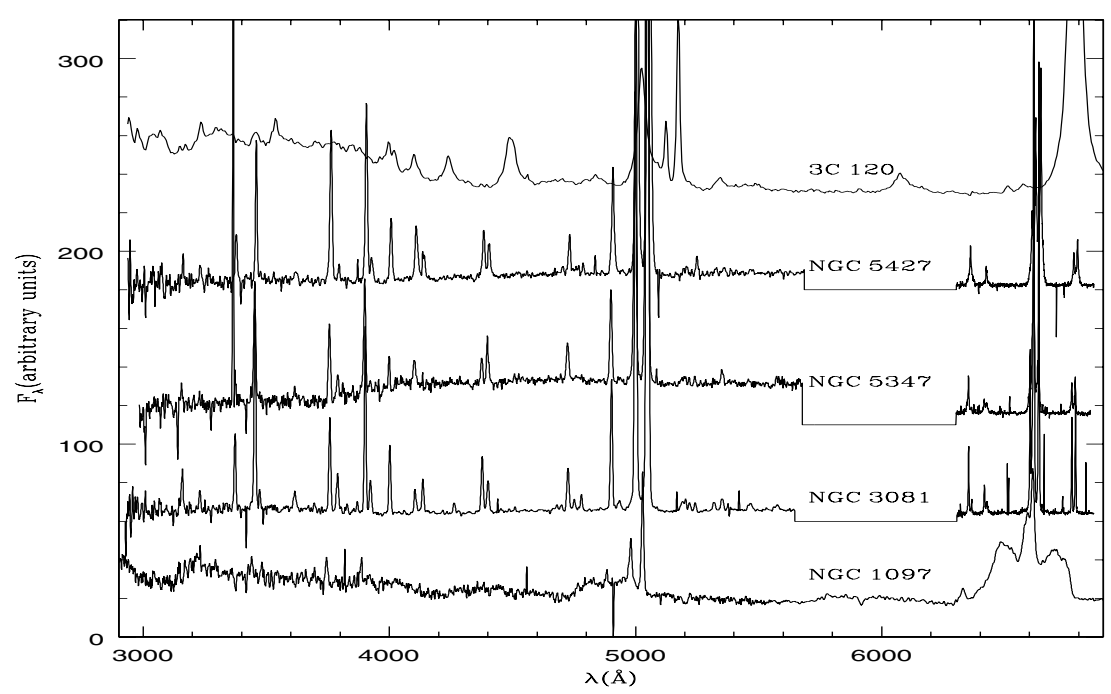

Figure 1. Sample of final spectra 